\title{
Application of Wireless Network in Hospital Information Construction
}

\author{
Yongrui Zhang ${ }^{1, a}$ and Jialin Gao ${ }^{1, b}$ \\ ${ }^{1}$ Department of Urology, The First Hospital of Jilin University, Jilin, China \\ azyr-912@163.com, bgjl@jlu.edu.cn
}

Keywords: Wireless network, Hospital information

\begin{abstract}
The rapid development and wide application of wireless network technology have brought unprecedented opportunities to the reform of medical and health services in China. Many hospitals in China have widely applied wireless network technology to informatization construction of hospitals, mainly including wireless room inspection, wireless infusion, wireless nursing, telemedicine, and warehouse management. This has the advantages of being flexible, convenient, effective, and reliable, which is conducive to the comprehensive integration of hospital data and information, reducing the work pressure of medical personnel, improving medical diagnosis and quality of care, and promoting the development of China's medical and health services. Wireless networks have made outstanding contributions in controlling medical defects and improving economic efficiency while improving work efficiency and reducing costs. However, there are still certain security risks in wireless networks. Only the safe and rational use of wireless network technology can ensure the hospital's information security.
\end{abstract}

\section{Introduction}

With the advent of the information age, wireless network technology has matured and created favorable conditions for hospital information construction. Clinical information construction has prompted the hospital to achieve paperless, filmless, and wireless [1]. This has greatly improved the efficiency of medical staff. The wireless network allows us to use any device to communicate with anyone in any place, anytime, simply, safely and stably. Under this trend, medical behaviors only stay in fixed time, fixed wards and clinics can no longer meet the needs of future development. The traditional wired network can not meet the need of communication anytime and anywhere. The application of wireless mobile network is unstoppable. Because the medical profession has certain particularity, it can make full use of the mobility, flexibility, expansibility and high transmission rate of wireless network technology to realize real-time patient information or diagnosis and treatment information. It makes up for the limitations of the cable network, highlights the technological advantages of the digital hospital and maximize the level of medical services with the core of patients. However, due to the particularity of the medical and health industry, the construction of hospital informatization also faces many new challenges.

\section{Hospital wireless network design}

The design of the hospital's wireless network structure in China is gradually improving. All of them adopt advanced wireless network technology, featuring large network capacity, high security, strong scalability, and high transmission quality. Taking the wireless network structure design of $\mathrm{Wu}$ Yue et al. [2] as an example, the transmission communication mode chooses the radio wave channel. The wireless AP (IEEE802.11n protocol standard) is installed in the various floors of the whole hospital, and the wireless signal is covered. The antenna feeder device is installed in the reasonable position of the hospital building to ensure the wider coverage of the wireless communication signal. The wireless network architecture of hospital can be divided into terminal layer, IP network layer and server cluster. The terminal layer is designed as location terminal, IP Phone, PDA and PC; The IP network layer is designed as $\mathrm{AP}, \mathrm{POE}$ switch, core switch and $\mathrm{AC}$; The server cluster is designed as location server, voice server, HIS / PACS and IMC intelligent management center. Access part: the wireless coverage area is connected to the POE switch by $\mathrm{AP}$, and then connected to the core switch or AC. Management part: in the network core layer, the most advanced third generation wireless network technology switches are used for unified management; Trapeze MX-200R wireless switch is used to manage and control AP. Wireless network management: mainly to enhance management, seamless security, and better planning of 
wireless network, but also to provide comprehensive control and optimization of AP, switch and controller. These parts together form an integrated smart mobile network.

\section{Advantages of wireless network application}

At present, most hospitals in China use wireless network technology to establish hospital information system, which is beneficial to improve management efficiency. With the development of information technology, the construction of patient centered clinical information system has become the application direction of wireless network, including nursing information system, doctor workstation system, operation anesthesia information system, inspection information system, radiology information system, medical image archiving and communication system. The application of wireless network in hospital information construction has significant advantages: 1) Network construction is fast and can connect to the network anytime and anywhere. Patients' case information can be seen in any ward. 2) Paperless transmission of data. The application of wireless network enables paperless transmission of data and information. It enables fast mobile query under wireless network and saves office supplies. 3) Paperless inspection. Medical workers connect laptops and other devices to wireless networks to quickly find patients' clinical data, such as medical history, treatment plans, medication details, and examination records. This can reduce the incidence of errors and omissions in the treatment of patients, effectively reduce the incidence of medical accidents, and improve the working efficiency of medical staff. 4) The monitoring facilities are networked, and hospitals, such as ICU, CCU and EICU, have higher requirements for monitoring devices. The monitoring equipment can realize the information management through the wireless network, which is helpful to realize the real-time of the monitoring work, observe the patient's vital signs and clinical changes in time, and ensure the safety of the patient's life.

\section{The specific application of wireless network in hospital information construction}

\subsection{Application of ward rounds}

After the establishment of wireless network in the ward, the problem of doctors suffering from network line was solved. Ward doctor workstations and nurse workstations are no longer controlled by the cable area. It can log in the doctor station and nurse station through the wireless network using the mobile workstation, move in the ward, and check the patient's hospitalization information and examination results and other monitoring information at any time . Doctors can give instructions at any time, and nurses can record patients' physical information in real time at the bedside. This has greatly improved the ability of doctors and nurses to track and implement patients' conditions and care. Some information such as physical examination can be entered into the computer. According to the change of the patient's condition, a doctor's order is drawn out, and the doctor's order is reused or the doctor's orders are made up again after the checkout, and repeated work and unnecessary mistakes are produced to obtain high efficiency and high quality bedside visit and nursing. Mobile medical workstation can query the implementation of doctor's orders in real time, better assist the whole life cycle tracking of medical advice, increase the monitoring and management of the work process and [3], and reduce medical disputes.

\subsection{Application of patient care}

In foreign countries, a considerable number of hospitals have canceled the ward care stations. The nursing information data is collected and recorded by setting up the wireless nursing system terminal at the bedside of the patient. The wireless network extends to the bedside of patients, which seems a small step in physical distance, but in fact, it can improve the efficiency and quality of nursing work. Moreover, the communication between medical staff and patients will be more convenient and frequent, which will help improve the affinity of nurses and effectively avoid accidents. For example, in order to enable patients to receive more comprehensive care, many hospitals' monitoring devices in ICU wards, EICU wards and other wards are connected to wireless network, which provides a guarantee for patients' life safety. In addition, based on wireless network technology to build a wireless nursing system, the system can accurately tell which patients need to measure body temperature and respiration, which patients need to measure weight and blood pressure, specific measurement time, operation time, discharge time and so on to inform the nursing staff. In this way, the work of nursing staff will be more orderly and the quality of work can be guaranteed.

\subsection{Application of outpatient infusion}

Mobile infusion information system based on wireless network technology is another important system widely applied in the field of hospital wireless network technology. Under normal circumstances, the number of outpatient infusions in hospitals is large, and the workload of medical staff will increase. In times of heavy business, some nurses may experience work errors due to minor negligence. The use of wireless networks will avoid these errors to a great extent. Because the system uses a PDA, a bar-code and wireless networking technology, nurses can check the bar-code on the medication at work based on the patient's identity. This improves the working efficiency of nurses, avoids mistakes, ensures the life safety of patients, and avoids some medical disputes and medical accidents. At the same time, the application of wireless call technology on the basis of wireless network can guarantee the response 
of nurses to a certain extent, improve the environment of the transfusion room and improve the satisfaction of the patients to the hospital.

\subsection{Application of warehouse management}

Bar-code identification is carried out for goods, medicine and documents in the hospital warehouse, and automatic identification is completed with bar-code reading equipment to improve the accuracy of identification operations[4]. The information construction of warehouse management is beneficial to improve the efficiency of warehouse operation, avoid errors in work, and improve the timeliness and accuracy of system data. In the management of fixed assets, hospitals are prone to problems such as idle assets, low efficiency, difficult inventory, loss of assets, and asset growth. The application of information-based warehouse management system and the strengthening of fixed assets management are effective ways to prevent the loss of assets, virtual increase and idle waste.

\subsection{Location tracking management}

Medical equipment is expensive, and any negligence in the maintenance process will increase the secondary investment of the hospital. Therefore, the regulation of medical devices, especially mobile medical devices, is crucial. The management of traditional medical equipment can only be solved by rules and regulations, which not only consumes manpower, but also has poor management. If we change to IT management and rely on wireless positioning system, this problem will be solved well. With the wireless positioning system, medical devices can be located through WLAN network only by putting special labels on medical devices. Managers can keep track of the location of medical devices from time to time with a single computer. In addition, for special patients or infants, they can also be located in real time through special wristbands.

\subsection{Application of specimen collection}

Automatic specimen collection system based on computer and wireless network communication technology has brought more benefits to hospitals. With automatic specimen collection system, when specimen collection and processing, medical staff can confirm the patient's identity and sample label information by hand held terminal. With the help of the RFID card reader, we can get all the data of the sample quickly, greatly reducing the probability of misdiagnosis and error, so as to ensure that the patient can get effective treatment.

\subsection{Application of remote diagnosis and treatment}

Telemedicine is a new form of medical treatment developed under the development of network technology. For some sudden diseases or miscellaneous diseases, the application of remote diagnosis and treatment can quickly control the development of the disease, cooperate with the medical institutions and formulate a difficult and complicated treatment plan. The hospital applies wireless network technology, multimedia technology and communication technology to medical work. The patient's disease information is transmitted through the network to realize the diagnosis and treatment of diseases under the network. Doctors can also communicate with patients online. It can also be discussed in the network system with other doctors in the network system. It is a low-cost method of diagnosis and treatment, which can effectively improve the level of social medical service.

\subsection{Application of call communication}

Hospitals can use the established wireless network to replace the traditional communication devices with the IP voice (VoIP) system. The realization of voice and data transmission in the same network can provide two-way voice and video communication, and realize voice and video data transmission in the network. The medical staff can receive the call of the patient through the handheld equipment, and can also see the call information in the hospital system, understand the patient's needs in time, so that the patient can be saved in time and special care.

\section{Summary}

With the rapid development of medical reform and the development and innovation of wireless technology, the application of WLAN in the medical industry around the world has become a trend. Hospitals are gradually realizing paperless and non film technology, and wireless network technology can achieve better results. The wireless network effectively overcomes the malpractice of the wired network, integrates all kinds of information data of the hospital, reduces the workload of the medical staff, improves the doctor's diagnosis and treatment level and the diagnostic efficiency, reduces the error rate, and avoids unnecessary medical accidents and medical disputes to some extent. To sum up, wireless network is the inevitable trend of hospital information development. In the future, we should strengthen the research on the application of wireless network technology, improve various wireless network systems, and truly play the role of hospital information system.

\section{References}

1. Niu Wei, Guo Shize, Wu Zhijun. Wireless LAN[M]. Beijing: People and Telecom Press, 2003: 9.

2. Wu Er, Xia Kaijian. Application and Design on the Wireless Network Technology in Hospital Information Construction [J]. Chinese Medical Equipment, 2013, 10(6): 41-43.

3. Chen Lijun, Chen Ying, Peng Weiping, et al. Application of PDA Wireless Network Nursing 
System in Clinical Nursing Work [J]. Chinese Journal of Practical Nursing, 2006, 22(5): 6-7.

4. Wang Lin. Application of Mobile and Wireless Technologies in Hospital Informatization
Construction [J]. Chinese Medical Equipment, 2013, 28(7): 79-80. 